\title{
A Agricultura Familiar no Município de Amambai-MS: uma análise dos desafios na comercialização da Associação de Agricultores Familiares
}

\author{
Fabricio Antonio Deffacci ${ }^{1}$ \\ Michelle Christina Castilho Ribeiro da Silva ${ }^{2}$
}

\begin{abstract}
RESUMO
O presente artigo visa analisar o processo de comercialização de alimentos entre o segmento da agricultura familiar e o mercado institucional, mediante o Programa de Aquisição de Alimentos (PAA) desenvolvido na Associação dos Agricultores Familiares de Amambai (Assafam), enfocando na sua modalidade Compra Institucional (CI), considerando o conceito de qualidade ampla como guia para a compreensão dos possíveis avanços e barreiras existentes e consequentemente a percepção dos fatores que contribuem com estes dois aspectos. O PAA e as chamadas públicas, apesar de apresentarem algumas barreiras e dificuldades para sua implantação e efetividade, ainda é visto como instrumentos de fortalecimento e de inclusão do segmento da agricultura familiar no mercado institucional. A proposta do PAA revela estar mais relacionada à qualidade ampla, podendo conduzir a construção de editais, mais abrangentes e eficazes em seus processos de comercialização. Compreende-se a necessidade de algumas estratégias de aproximação e diálogo com as organizações de agricultores familiares com o intuito de superar dificuldades existentes no processo, aprimorando assim a relação de compra e venda entre o segmento da agricultura familiar e os órgãos públicos.
\end{abstract}

Palavras-Chave: controle de qualidade; desperdício; associação; agricultura familiar.

\section{Family Farming in the Municipality of Amambai-MS, Brazil: an analysis of the commercialization challenges of the Family Farmers Association (ASSAFAM)}

\begin{abstract}
This article aims to analyze the process of food commercialization between the family farming segment and the institutional market, through the Food Acquisition Program (PAA) developed at the Amambai Family Farmers Association (Assafam), focusing on its Institutional Purchasing modality. (CI), considering the concept of broad quality as a guide for understanding the possible advances and existing barriers and consequently the perception of the factors that contribute to these two aspects. The PAA and the public calls, despite presenting some barriers and difficulties for their implementation and effectiveness, are still seen as instruments for strengthening and including the family farming segment in the institutional market. The PAA proposal reveals to be more related to the broad quality and may lead to the construction of edicts, more comprehensive and effective in its commercialization processes. It is understood the need for some strategies of approach and dialogue with family farmers organizations in order to overcome existing difficulties in the process, thus improving the buying and selling relationship between the family farming segment and public agencies.
\end{abstract}

Keywords: quality control; waste; association; family farming.

\section{INTRODUÇÃO}

Este trabalho se propôs a investigar as dificuldades encontradas pelos agricultores familiares pertencentes ao município de Amambai/MS para a comercialização dos alimentos produzidos pela na Associação dos Agricultores Familiares de Amambai (ASSAFAM). Busca-

\footnotetext{
${ }^{1}$ Doutor em Ciências Sociais. Professor do Programa de Pós-Graduação em Desenvolvimento Regional e de Sistemas Produtivos da Universidade Estadual de Mato Grosso do Sul (UEMS). fabricio.deffacci@ gmail.com

${ }^{2}$ Mestrando do Programa de Pós-Graduação em Desenvolvimento Regional e Sistemas Produtivos (PPGDRS) pela Universidade Estadual do Mato Grosso do Sul (UEMS), Unidade de Ponta Porã-MS.
} 
se por meio dessa pesquisa compreender os aspectos que estão relacionados ao processo de comercialização de alimentos entre o segmento da agricultura familiar e o mercado institucional, dentro do Programa de Aquisição de Alimentos (PAA), visando identificar as barreiras e procurar soluções para aquelas já existentes no município.

A ASSAFAM foi fundada em 2014 formada inicialmente por 23 agricultores familiares, em 2016 a associação era responsável pelo fornecimento de parte das verduras, legumes e hortaliças servidos para os alunos das escolas públicas do município. Com a assistência da Agência de Desenvolvimento Agrário e Extensão Rural (AGRAER), órgão do governo do Estado, os produtores da Associação de Agricultores Familiares de Amambai (ASSAFAM) em 2017 venderam seus produtos para o Exército Brasileiro, através do $17^{\circ} \mathrm{RCMEC}$ - Regimento de Cavalaria Mecanizada, do município de Amambai.

O fechamento do negócio ocorreu por meio da Chamada Pública nº 01/2017, essa se apresentou como um novo mercado que os agricultores do município de Amambai/MS que passaram a ter para comercializar os alimentos que são cultivados dentro dos seus sítios "É uma atividade rentável em que posso sustentar a minha família”, afirma a agricultora Zeila Moreira que reside em uma das áreas rurais de cultivo, a Vila Santo Antônio (AGRAER, 2017).

De acordo com a AGRAER, este contrato é outra maneira do mercado favorecer a agricultura familiar no município, tendo em vista que os produtores já conquistaram uma parte do mercado local e ainda contam com a venda de alimentos para a rede pública de ensino através do Programa Nacional de Alimentação Escolar (PNAE).

Diante desse cenário de produção e comercialização, será abordado o conceito de qualidade destacando que esta não está vinculada somente às propriedades do próprio alimento, mas está também relacionada com os modos adotados para que estas propriedades sejam alcançadas. (MORRIS, YOUNG; 2000). Deste modo, se o conceito de qualidade for visto pela ótica mais diversificada de aspectos, pode possibilitar a maior eficácia dentro dos processos de comercialização, no caso das chamadas públicas.

No que se refere à produção de alimentos provenientes da agricultura familiar, existem algumas barreiras, como aquelas que dizem respeito às normas sanitárias, que dificultam a comercialização, ultrapassando assim as questões de gestão, e condicionam alguns estabelecimentos produtores a atuar no setor informal. Wilkinson e Mior (1999), afirmam que este se difere do ilegal, uma vez que os produtos advindos do setor informal não são proibidos como no caso de situações relacionadas ao contrabando, por exemplo, mas relaciona-se a uma atividade em que os processos de produção não se adequam nos padrões de regulação vigentes. 
Ainda que existam normas mais próximas da realidade produtiva em pequena e média escala, ocorrem problemas relacionados aos métodos de fiscalização, pautados em resoluções voltadas à larga escala de produção, a exemplo da Resolução n ${ }^{\circ}$ 275, de 21 de outubro de 2002, da Agência Nacional de Vigilância Sanitária (ANVISA), dificultando ,muitas vezes, o crescimento dos setores produtivos de pequena escala, e a inserção de famílias agricultoras que processam alimentos de maneira artesanal, no mercado de alimentos. Isto ocorre, pois, a utilização destas normas de maneira descontextualizada, ou seja, para produção em pequena e média escala, impossibilita que estes produtores se adéquem às exigências prescritas na lei, mantendo-se às margens do mercado de alimentos (ISPN, 2012).

Portanto, não é suficiente a construção de normas específicas, adequadas à realidade produtiva, é necessário promover a sensibilização dos agentes de fiscalização, para que possam auxiliar na qualificação continuada dos produtores, objetivando a legalização da produção e consequente inserção no mercado. (ISPN, 2012). Não se trata, pois, apenas de flexibilizar as normas, mas sim em contextualizá-las, de modo que sua elaboração ocorra mediada pela realidade produtiva e pelos reais riscos de contaminação provenientes da pequena e/ou média escala de produção.

Por essa razão, a presente pesquisa torna-se relevante para o campo do desenvolvimento regional e local, tendo em vista que a alimentação da população brasileira possui uma significativa participação da agricultura familiar, como base produtora, responsável pelo abastecimento alimentar do país, fazendo-se sempre presente na conformação da nossa sociedade. No entanto, no Brasil esse segmento requer maiores investimentos e cuidados estratégicos e administrativos para que a participação seja cada vez mais fortalecida, havendo a disponibilidade de alimentos para a população (BURLANDY, 2009).

\section{FUNDAMENTAÇÃO TEÓRICA}

Em meio às questões que envolvem um processo de comercialização entre agricultores familiares e órgãos públicos, algumas são destacadas aqui, relativas às concepções sobre qualidade que podem estar presentes e que norteiam a elaboração de editais voltados à aquisição de gêneros alimentícios. A visão que os indivíduos envolvidos na elaboração dos editais possuem sobre o conceito de qualidade, bem como, o conhecimento que acessam e a visão de mundo, afetam as escolhas dos aspectos que se farão presentes nos editais de chamada pública. 
A compreensão do termo qualidade faz-se necessária, uma vez que existe uma procura constante por parte do público consumidor por alimentos e produtos que ofereçam qualidade. Conforme destacam Schneider e Ferrari (2015), embora o padrão predominante ainda seja aquele baseado no consumo de alimentos/produtos produzidos em grande escala, há entre os países desenvolvidos e os em desenvolvimento, sinais de que está aumentando a busca pelos consumidores por alimentos com características mais saudáveis e com a presença de uma qualidade diferenciada.

Portanto partiu-se da ideia de que o termo qualidade pode ser entendido de perspectivas variadas e, a partir disto, questiona-se qual o impacto que as diversas visões podem causar em diferentes tipos de produtores de alimentos e consequentemente as interferências geradas nos processos produtivos e de comercialização.

No que se refere aos termos técnico-científicos, as normas regulamentadoras vigentes vinculadas à prática da vigilância sanitária, geralmente, são construídas com uma visão de qualidade mais higienista que valoriza aspectos como os físico-químicos e microbiológicos (CRUZ, 2012), sendo que apesar da importância destes aspectos, como trazido por Poulain (2004, pg. 110-111) "os conhecimentos científicos podem ser colocados a serviço do respeito e do melhoramento de um modelo alimentar, tentando compreender as condições de redução dos riscos sanitários, em respeito às dimensões gustativas e simbólicas”, por exemplo. Deste modo, considerar a qualidade dos alimentos somente pelo olhar técnico-científico, que contempla a inocuidade como sinônimo de qualidade, pode conduzir produtores de alimentos que utilizam das práticas mais tradicionais ou que produzem em menor escala, a atuar à margem do setor produtivo de alimentos. (CRUZ, 2009).

Evidencia-se que o conceito de qualidade que será abordado no presente artigo, não pretende se limitar apenas ao padrão de qualidade solicitado aos alimentos, a exemplo das características sensoriais e microbiológicas, mas se relacionará, ainda, aos modos de produção, às categorias produtoras, às tradições alimentares, à cultura, dentre outros aspectos, considerados mais externos, e que giram ao redor de um conceito mais abrangente de qualidade.

Portanto no que se refere às normas para a produção e comercialização de alimentos que podem ser utilizadas nos editais de chamadas públicas, estas são formuladas e divulgadas por meio da Agência Nacional de Vigilância Sanitária (ANVISA) relacionada ao Ministério da Saúde ou por meio do Ministério da Agricultura, Pecuária e Abastecimento (MAPA), que indicam quais são os padrões, como os de qualidade, higiene e conservação, que os alimentos 
devem apresentar, auxiliando nas descrições dos alimentos presentes em editais de aquisição para os órgãos públicos. (AGUIAR; CALIL, 2016).

Deste modo é possível encontrarmos disponível os Padrões de Identidade e Qualidade (PIQ's) que são instrumentos que possuem um conjunto de características que identificam e qualificam um produto/alimento. Estes padrões definem características mínimas aceitáveis sobre as variadas categorias de alimentos, indicando se os alimentos poderão ser comercializados e consumidos (BRASIL, 1993b), sendo cada categoria de alimento analisada de acordo com uma legislação específica.

Cabe destacarmos que de modo geral, as normas utilizadas como referência conceituam a qualidade de um alimento basicamente a partir dos aspectos sensoriais, físico-químicos e microbiológicos, apesar da importância destes aspectos serem controlados durante a produção dos alimentos para que sejam seguros para o consumo, acredita-se que o conceito de qualidade pode incluir também outras questões igualmente importantes, principalmente quando se pensa em inclusão de produtores e produtos no mercado de alimentos.

\section{CONCEITO DE QUALIDADE: PRESCRITIVO E NORMATIVO}

O embate sobre o conceito de qualidade pode estar diretamente ligado à questão relacionada às legislações existentes e as maneiras em que estas são desenvolvidas e aplicadas, pois para analisarmos as perspectivas de qualidade empregadas em determinados processos de compra e venda, como em editais de chamadas públicas, por exemplo, deve-se pensar sobre as visões que orientam a construção de normas que buscam a inserção dos produtores no mercado e os controles empregados frente as fiscalizações. Assim sendo abordaremos aqui a influência que a definição de qualidade pode ter na construção de normas regulamentadoras e fiscalizadoras, pois é neste momento que surgem algumas barreiras.

Por essa razão destaca-se que os critérios de qualidade são importantes elementos para a produção de alimentos, porém nota-se que para pequenos e médios produtores, há certa dificuldade em atender as demandas, principalmente as relacionadas com a adequação sanitária, como alguns estudos já apontaram. (PLEIN; FILIPPI, 2012; CINTRÃO, 2014).

Portanto salientaremos dois tipos principais de controle de qualidade sendo estes o Prescritivo e Normativo, o primeiro considera a legislação sanitária vigente, suas fiscalizações tendem a serem realizadas com base no controle de qualidade prescritivo. Neste tipo de controle, ocorre a identificação do problema relacionado à qualidade por meio de análises, 
como a laboratorial, que indicam se um alimento será considerado adequado ou não para o consumo humano.

Como consequência da fiscalização do controle prescritivo, no caso da não aptidão do alimento, este será descartado e a produção voltará a ser normalizada após a regularização das questões impeditivas apontadas pelo laudo técnico. Caso o local apresente irregularidade de produção, o responsável pelo estabelecimento sofrerá impedimentos para dar continuidade à produção ou receberá punições por meio de multas. (SILVEIRA; HEINZ, 2005).

Deste modo nesse tipo de controle, as fiscalizações assumem caráter punitivo e o agente responsável pelo processo não age como um educador. Evidencia-se que um agente educador é aquele que busca, em conjunto com os produtores, soluções para resolver as inconformidades por meio das trocas de conhecimentos e participação ativa (SILVEIRA; HEINZ, 2005), considerando perigos e pontos críticos de controle. Assim, o controle de qualidade prescritivo irá basear-se, além do caráter punitivo, em um caráter corretivo. (SILVEIRA; HEINZ, 2005).

No que se refere ao modo de controle normativo diferentemente do prescritivo este controle de qualidade é caracterizado como um método de caráter processual e participativo, com a troca de saberes e conhecimentos entre todos os envolvidos no processo produtivo (técnicos, produtores, agentes fiscalizadores, dentre outros), além de considerar que as normas que serão utilizadas deverão ser definidas com base na realidade existente nos estabelecimentos.

Salienta-se aqui que esta afirmação não significa flexibilizar normas e deixar de garantir um alimento seguro, mas sim em existir um controle por meio de legislações produzidas a partir da realidade produtiva dos variados tipos de locais. Deste modo, para que este tipo de controle aconteça, deve haver uma ação educativa, de modo a ocorrer uma apreensão da realidade e dos saberes nela envolvidos, culminando, por exemplo, na construção de normas, procedimentos e em ações baseadas em uma realidade específica (SILVEIRA; HEINZ, 2005). Para facilitar a diferenciação dos dois tipos de controle de qualidade no quadro 2 abaixo:

\begin{tabular}{clc}
\hline \multicolumn{1}{c}{ Controle de qualidade prescritivo } & \multicolumn{2}{c}{ Controle de qualidade normativo } \\
\hline Fiscalizações com caráter punitivo e corretivo & $\begin{array}{l}\text { Fiscalizações com caráter processual e } \\
\text { participativo }\end{array}$ \\
\hline Agente não age como um educador & $\begin{array}{l}\text { Agente age como um educador - há respeito aos } \\
\text { saberes locais }\end{array}$ \\
\hline $\begin{array}{c}\text { Qualidade ótima - a ótica está mais voltada } \\
\text { para as questões sanitárias e legais: }\end{array}$ & $\begin{array}{l}\text { Qualidade ampla - considera a qualidade ótima } \\
\text { - Alimento associado à doença; }\end{array}$ & $\begin{array}{l}\text { Qualidade sanitária ou higiênica, } \\
\text { nutricional, organoléptica (sensorial), } \\
\text { facilidade de uso, regulamentar; } \\
\text { Estrutura e manipuladores como } \\
\text { meios de contaminação }\end{array}$ \\
& $\begin{array}{l}\text { Social, cultural, ambiental (ecológico)e } \\
\text { aparência }\end{array}$ \\
\hline
\end{tabular}

Fonte: Adaptado de Prezotto (2002); Silveira; Heinz (2005). 
No entanto é importante destacarmos, que as normas e os procedimentos de regulação devem ser construídos em conformidade com as escalas de produção, permitindo a atuação legal, tanto em largas escalas de produção, quanto em pequenas e médias escalas de produção. Desta maneira, as normas sanitárias específicas buscam como objetivo a produção segura do alimento e a inserção de diferentes segmentos produtivos no mercado de alimentos (PREZOTTO, 1999).

\section{METODOLOGIA}

A metodologia que norteia o presente artigo é uma pesquisa-ação. Conforme indica Thiollent (1985) caracteriza-se por se tratar de uma pesquisa definida por pesquisa-ação, deste modo quando a pesquisa demonstra que realmente há uma ação por parte das pessoas envolvidas no processo investigativo, a partir do desenvolvimento de um projeto de ação social ou da solução de problemas coletivos e esta centrada no agir participativo e na ideologia de ação coletiva. Para alcançar o objetivo proposto na pesquisa-ação no que se refere em estabelecer uma relação entre o conhecimento e ação, entre pesquisadores e pessoas envolvidas na situação investigada e com as suas realidades. Logo, a pesquisa-ação exige uma estrutura de relação entre os pesquisadores e pessoas envolvidas no estudo da realidade do tipo participativo/coletivo.

A pesquisa partirá de um levantamento bibliográfico desenvolvido a partir de trabalhos já desenvolvidos acerca do tema os desafios e oportunidades para a agricultura familiar frente às compras públicas além destes trabalhos que auxiliaram no desenvolvimento da pesquisa, a proposta também contará com a base histórica acerca de Amambai. Portanto é importante destacar que no decorrer do processo da pesquisa bibliográfica é importante que o pesquisador faça anotações e fichamentos sobre os conteúdos considerados mais importantes, e que possivelmente serão utilizadas como fundamentação teórica em sua pesquisa. (FONSECA, 2002, p. 32)

Após a isso, será realizada a pesquisa de campo onde será realizada visitas na Associação dos Agricultores Familiares de Amambai (ASSAFAM), onde será possível conversar com os associados e por meio de uma entrevista semiestruturada identificar as oportunidades e os desafios enfrentados pelas Associações levando em consideração o controle de qualidade e principalmente o quesito desperdício. Em momento posterior, com o subsídio 
de outra pesquisa responsável por articular os dados obtidos até o momento, será proposto um projeto que venha a auxiliar a ASSAFAM a diminuir o desperdício e que possa vir a contribuir gerando lucro e oportunidade, beneficiando deste modo os agricultores associados e o município de Amambai/MS.

\section{O PROBLEMA DO DESPERDÍCIO}

Existe outro fator que deve ser considerado na definição de qualidade, diz respeito ao desperdício de alimentos, devido à imposição dos padrões existentes, que classificam um alimento como de qualidade ou não. Estes estão relacionados principalmente aos aspectos estéticos dos alimentos, não interferindo, muitas vezes, em outros aspectos de qualidade. Desta forma, existe a necessidade em se pensar na qualidade do alimento além da sua característica estética, já que alimentos considerados disformes em relação a sua aparência e sob a ótica das normas de classificação, são rejeitados e assim o desperdício surge como fator negativo. (SIROTA; ANJOS; COSTA, 2016). Veja na tabela 1 a Produção Média Comercializada pela ASSAFAM:

Tabela 1 - produção média comercializada da ASSAFAM

\begin{tabular}{|l|l|l|l|}
\hline \multicolumn{5}{|c|}{ PRODUÇ̃̃O MÉDIA COMERCIALIZADA DA ASSAFAM } \\
\hline Produto & Quantidade (Kg/Ano) & Valor Unitário - R\$ (Kg) & Valor Total - R\$ \\
\hline Mandioca & 14.000 & 2,82 & $39.480,00$ \\
\hline Cenoura & 14.000 & 3,26 & $45.640,00$ \\
\hline Chuchu & 400 & 2,83 & $1.132,00$ \\
\hline Beterraba & 12.000 & 4,09 & $49.080,00$ \\
\hline Batata-doce & 15.000 & 2,92 & $43.800,00$ \\
\hline Abobrinha & 1.200 & 4,26 & $5.112,00$ \\
\hline Cabotiá & 500 & 2,65 & $1.325,00$ \\
\hline Cebola & 4.000 & 7,60 & $30.400,00$ \\
\hline Alho & 100 & 12,00 & $1.200,00$ \\
\hline Tomate cereja & 200 & 4,00 & 800,00 \\
\hline Quiabo & 300 & 3,00 & 900,00 \\
\hline Jiló & 300 & 2,50 & 750,00 \\
\hline Vagem & 400 & 4,00 & $1.600,00$ \\
\hline Repolho & 3.000 & 2,91 & $8.730,00$ \\
\hline Banana & 2.000 & 2,00 & $4.000,00$ \\
\hline
\end{tabular}




\begin{tabular}{|l|l|l|l|}
\hline Morango & 2.000 & 19,30 & $38.600,00$ \\
\hline Milho verde & 3.000 & 6,39 & $19.170,00$ \\
\hline Alface & 30.000 & 2,50 & $75.000,00$ \\
\hline Acelga & 1.000 & 4,80 & $48.500,00$ \\
\hline Brócolis & 700 & 5,28 & $3.696,00$ \\
\hline Couve & 4.000 & 3,00 & $12.000,00$ \\
\hline Couve-flor & 2.000 & 5,00 & $10.000,00$ \\
\hline Almeirão & 600 & 3,00 & $1.800,00$ \\
\hline Rúcula & 600 & 3,00 & $1.800,00$ \\
\hline Cheiro verde & 7.000 & 2,16 & $15.120,00$ \\
\hline TOTAL & & & $459.635,00$ \\
\hline
\end{tabular}

Estima-se que são desperdiçadas anualmente em todo o mundo, aproximadamente 1,3 milhão (um milhão e trezentos mil) toneladas de alimentos, representando um terço dos alimentos produzidos para o consumo humano. Nos países em desenvolvimento, as perdas (cerca de 40\%) ocorrem nas etapas de pós colheita e processamento e, de um modo geral, os alimentos que sofrem maiores desperdícios (cerca de 45\%) pertencem aos grupos das frutas, hortaliças, raízes e tubérculos. Veja abaixo na tabela 2 referente ao desperdício/perda da produção final da ASSAFAM:

Tabela 2 - desperdício da produção da ASSAFAM

\begin{tabular}{|l|l|l|l|}
\hline \multicolumn{5}{|c|}{ DISPERDÍCIO/PERDAS DA PRODUÇÃO FINAL DA ASSAFAM } \\
\hline Produto & Quantidade (Kg/Ano) & Valor Unitário - R\$ (Kg) & Valor Total - R\$ \\
\hline Mandioca & 6.000 & 2,82 & $16.920,00$ \\
\hline Cenoura & 6.000 & 3,26 & $19.560,00$ \\
\hline Chuchu & 400 & 2,83 & $1.132,00$ \\
\hline Beterraba & 8.000 & 4,09 & $32.720,00$ \\
\hline Batata-doce & 15.000 & 2,92 & $43.800,00$ \\
\hline Abobrinha & 800 & 4,26 & $3.408,00$ \\
\hline Cabotiá & 200 & 2,65 & 530,00 \\
\hline Cebola & 2.000 & 7,60 & $15.200,00$ \\
\hline Alho & 400 & 12,00 & $4.800,00$ \\
\hline Tomate cereja & 500 & 4,00 & $2.000,00$ \\
\hline Quiabo & 200 & 3,00 & 600,00 \\
\hline Jiló & 200 & 2,50 & 500,00 \\
\hline Vagem & 100 & 4,00 & $4.000,00$ \\
\hline Repolho & 2.000 & 2,91 & $5.820,00$ \\
\hline
\end{tabular}




\begin{tabular}{|l|l|l|l|}
\hline Banana & 2.000 & 2,00 & $4.000,00$ \\
\hline Morango & 1.000 & 19,30 & $19.300,00$ \\
\hline Milho verde & 2.800 & 6,39 & $17.892,00$ \\
\hline Alface & 10.000 & 2,50 & $25.000,00$ \\
\hline Acelga & 350 & 4,80 & $1.680,00$ \\
\hline Brócolis & 700 & 5,28 & $3.696,00$ \\
\hline Couve & 4.000 & 3,00 & $12.000,00$ \\
\hline Couve-flor & 2.200 & 5,00 & $11.000,00$ \\
\hline Almeirão & 400 & 3,00 & $1.200,00$ \\
\hline Rúcula & 400 & 3,00 & $1.200,00$ \\
\hline Cheiro verde & 3.000 & 2,16 & $6.480,00$ \\
\hline TOTAL & & & $254.438,00$ \\
\hline
\end{tabular}

No total, a ASSAFAM apresenta um desperdício de aproximadamente $\mathrm{R} \$ 254.438,00$ por ano. Estes dados mostram que o desperdício nestas etapas se refere possivelmente à seleção, que já ocorre no campo, de alimentos considerados inadequados frente à visão dos padrões de qualidade (muitas vezes os produtores fazem esta seleção de alimentos, já que estes não serão aceitos nos locais de revenda), como ainda a falta de cuidado durante o manuseio dos alimentos e transporte, falta de condições adequadas na exposição dos alimentos, maus hábitos dos consumidores no momento da escolha dos alimentos para compra, dentre outros.

Destaca-se ainda que as perdas não estão relacionadas somente a questão econômica, mas também a questão ambiental, já que há perdas dos recursos naturais como uso da água e solo, com consequências climáticas. Dentre as perdas mundiais, $6 \%$ ocorrem na América Latina e Caribe, sendo que esta quantidade de alimentos poderia alimentar 300 milhões de pessoas. Por outro lado, nestas mesmas regiões, contabilizou-se que entre os anos de 2014 e 2016, aproximadamente 34 milhões de pessoas estavam em condições de subalimentação. (FAO, 2011; FAO, 2015; FAO,2017).

Assim, a partir dos dados acima sobre desperdício, busca-se atentar para a forma como os padrões de qualidade são construídos e o impacto que estes podem causar na Associação dos Agricultores Familiares de Amambai (ASSAFAM) e propriamente ao município em si. Ainda, reflete-se sobre quais aspectos realmente podem ser considerados e contribuem fortemente para a segurança alimentar e nutricional dos indivíduos, já que existe grande desperdício de alimentos, tendo como causa também os padrões de qualidade que devem ser seguidos, e a considerável parcela de indivíduos que estão em situação de subalimentação. 
Portanto, por meio da visão apresentada, acerca dos tipos de controle de qualidade este servirá como eixo norteador, cabendo a este envolver outros aspectos como questões ética e econômicas, fomentando discussões a respeito da questão por meio dos resultados levantados nessa pesquisa. Por certo, a pesquisa terá continuidade para se buscar um planejamento estratégico e coletivo no sentido de conceber procedimentos adequados para o aproveitamento dos produtos desperdiçados.

\section{CONSIDERAÇÕES FINAIS}

Este é o momento de pontuar algumas perspectivas enunciadas ao longo da realização da pesquisa. Dizer, porém, que um trabalho desta natureza e complexidade teve seu término, é justamente incoerente com o trabalho da pesquisa científica.

O presente artigo apresentou as percepções acerca da Associação dos Agricultores Familiares de Amambai (ASSAFAM), participantes dos processos de elaboração das chamadas públicas ou das chamadas públicas para a aquisição de gêneros alimentícios promovidos por uma instituição pública, possibilitando deste modo a melhor compreensão da relação existente entre agricultores familiares e órgãos públicos, no que se refere ao processo de comercialização de alimentos dentro do PAA modalidade Compra Institucional.

Associado a este contexto, procurou-se entender de que modo a percepção sobre qualidade pode interferir na seleção de propostas elaboradas pelas organizações de seleção da agricultura familiar visando a inserção no mercado institucional.

\section{REFERÊNCIAS}

AGUIAR, J.A.; CALIL, R.M. Análise e avaliação das especificações dos alimentos contidas em editais de chamadas públicas do PNAE. Revista Visa em Debate, Rio de Janeiro, v.4, n.2, p. 79-87, 2016. Disponível em:

https://visaemdebate.incqs.fiocruz.br/index.php/visaemdebate/article/view/580/3 9

BRASIL. Lei ${ }^{\circ}$ 8.666, de 21 de junho de 1993. Regulamenta o art. 37, inciso XXI, da Constituição Federal, institui normas para licitações e contratos da Administração Pública e dá outras providências. Diário Oficial da União, Brasília, DF, 22 jun. 1993a. Seção 1, p. 8269.

BURLANDY, L. A construção da política de segurança alimentar e nutricional no Brasil: estratégias e desafios para a promoção da intersetorialidade no âmbito federal de governo. Ciência e Saúde Coletiva, v. 14, n.3, p.851-860, 2009. 
CRUZ, F.T. da. Qualidade dos alimentos e escalas de produção: em defesa de critérios e normas para legitimação dos produtos artesanais/tradicionais. Trabalho apresentado no grupo de pesquisas: Agricultura familiar e Ruralidade. SOBER - Sociedade Brasileira de Economia, Administração e Sociologia Rural. Porto Alegre, 2009.

CRUZ, F.T. da. Produtores, consumidores e valorização de produtos tradicionais: um estudo sobre qualidade de alimentos a partir do caso do queijo serrano dos Campos de Cima da Cerra - RS. 292 f. Tese (Doutorado em Desenvolvimento Rural) - Universidade Federal do Rio Grande do Sul, Porto Alegre, 2012.

FOOD AND AGRICULTURE ORGANIZATION OF THE UNITED NATIONS (FAO). Global food losses and food waste - Extent, causes and prevention. Roma, 2011. Disponível em: <http://www.fao.org/docrep/014/mb060e/mb060e.pdf>.

FOOD AND AGRICULTURE ORGANIZATION OF THE UNITED NATIONS (FAO). Ano

Internacional da Agricultura Familiar. 2014. Disponível em: $<$ http://www.fao.org/3/cas281o.pdf $>$.

FOOD AND AGRICULTURE ORGANIZATION OF THE UNITED NATIONS (FAO). EI estado de la inseguridad alimentaria en el mundo. Cumplimiento de los objetivos internacionales para 2015 en relación con el hambre: balance de los desiguales progresos. Roma, 2015. Disponível em: 〈http://www.fao.org/3/ai4646s. pdf.>

FONSECA, J. J. S. Metodologia da pesquisa científica. Fortaleza: UEC, 2002.

INSTITUTO SOCIEDADE, POPULAÇÃO E NATUREZA (ISPN). Oficina Normas sanitárias para alimentos de produção artesanal, familiar e comunitária. Relatório final. Brasília, 2012. Disponível em:

<http://formsus.datasus.gov.br/novoimgarq/12601/1801884_134647.pdf>.

MORRIS, C.; YOUNG, C. 'Seed to shelf', 'teat to table', 'barley to beer' and 'womb to tomb': discourses of food quality and quality assurance schemes in the UK. Journal of Rural Studies, v.16, n. 1, p. 103-115, 2000. Disponível em:

<http://www.sciencedirect.com/science/article/pii/S0743016799000443>.

PLEIN, C.; FILIPPI, E. E. O Programa Aquisição de Alimentos da Agricultura Familiar (PAA): geração de renda e segurança alimentar. Revista Faz Ciência, Francisco Beltrão, v.14, n.19, p. 63-86, 2012. Disponível em: <http://erevista.

unioeste.br/index.php/fazciencia/article/viewArticle/8027>.

POULAIN, J. Sociologias da alimentação: os comedores e o espaço social alimentar. $1^{\mathrm{a}}$ ed. Florianópolis: Editora da UFSC, 2004. 
PREZOTTO, L.L. A agroindústria rural de pequeno porte e o seu ambiente institucional relativo à legislação sanitária. Dissertação (Mestrado em Agrossistemas) - Centro de Ciências Agrárias, Universidade Federal de Santa Catarina, Florianópolis, 1999.

SILVEIRA, P. R. C.; HEINZ, C. U. Controle de qualidade normativo e qualidade ampla: princípios para reestruturação e qualificação da produção artesanal de alimentos. Trabalho apresentado no $1^{\circ}$ Congresso Internacional de Desenvolvimento Rural e Agroindústria Familiar. São Luiz Gonzaga, 2005.

SIROTA, V.G.R; ANJOS, M.C.R.dos; COSTA, I.B.da. Padronização da qualidade de alimentos: uma ameaça à soberania alimentar. Espacio Regional: Revista de Estudios Sociales, Osorno - Chile, v.1, n.13, p. 37-48, 2016.

SCHOTTZ, V.; CINTRAO, R. P.; SANTOS, R. M. dos. Convergências entre a Política Nacional de SAN e a construção de normas sanitárias para produtos da Agricultura Familiar. Revista Visa em debate, Rio de Janeiro, v.2, n.4, p. 115-123, 2014. Disponível em: $<$ https://visaemdebate.incqs.fiocruz.br/index.php/visaemdebate/article/view/461/175.

SCHNEIDER, S; FERRARI, D.L. Cadeias curtas, cooperação e produtos de qualidade na agricultura familiar - o processo de relocalização da produção agroalimentar em Santa Catarina. Revista Organizações Rurais e Agroindustriais, Lavras, v. 17, n. 1, p. 56-71, 2015. Disponível em: <http://revista.dae.ufla.br/index.php/ora/article/view/949/0>.

THIOLLENT, Michel. Metodologia da Pesquisa-Ação. São Paulo: Cortez,1985.

WILKINSON, J.; MIOR, L.C. Setor informal, produção familiar e pequena agroindústria: interfaces. Estudos, Sociedade e Agricultura, Rio de Janeiro, n.13, p.29-45, 1999. Disponível em:http://r1.ufrrj.br/esa/V2/ojs/index.php/esa/article/view/159/155. 